Azizi V., Memarian H.

( School of Mining Engineering, University of Tehran, Iran )

\title{
PREDICTION OF MECHANICAL PARAMETERS OF ROCK, USING SHEAR WAVE TRAVEL TIME
}

Different methods have been used for downhole measuring of formation's shear wave travel time. These include visual inspection of full wave train recordings (e.g. variable density microseismogram), monitoring the amplitude characteristic of acoustic shear wave in selected gate(s), analysis of digital sonic data and comparison of compressional and shear wave transient time at various transmitter-receiver spacing and defining some experimental correlation between them. All these techniques have limited application, especially in the case of formations with complex lithology (combination of sandstone, dolomite, limestone, and anhydrite) and fluid content. This article presents an analytical approach for lowering the level of uncertainty in using above mentioned methods. The main objectives of present research are: reducing the level of uncertainty for different parameters and formation fluid contents, overcoming some of the limitations of existing methods and predicting mechanical parameters of drilled rocks. This has been done by utilizing the standard compressional travel time in conjunction with supplementary none-acoustic $\log$ data and petrophysical full logs interpretation results. Presented method can estimate shear wave travel time and some rock mechanical parameters with high accuracy for different lithology, especially for limestone-sandstone or limestone-dolomite-shale sequences.

Keywords: Shear wave travel time, Sonic log data, Petrophysical data, Mechanical properties of rock. 